\title{
Areal Surface Texture Parameters for Copper/Glass Plating Adhesion Characteristics
}

\author{
Baofeng $\mathrm{He}^{1}$, D. Patrick Webb ${ }^{2}$, Jon Petzing ${ }^{2 *}$ \\ ${ }^{1}$ Beijing Engineering Research Center of Precision Measurement Technology and Instruments, Faculty of Materials and \\ manufacturing, Beijing University of Technology, 100 Ping Le Yuan, Chaoyang District, Beijing 100124, China \\ ${ }^{2}$ Wolfson School of Mechanical and Manufacturing Engineering, Loughborough University, Loughborough, LE11 $3 T U$, UK \\ J.Petzing@lboro.ac.uk
}

\begin{abstract}
Glass as an alternative printed circuit board material and interposer has been investigated for use in the micro-electronics industry. Electroless copper plating is used to provide the conductive layer, but there is limited understanding of how the surface topography of the glass substrate affects the copper/glass bonding strength exhibited in the current literature. A laser ablation technique was used to prepare glass surfaces with micro-scale structured features in this study, and these features were characterized quantitatively using areal surface texture parameters. The copper/glass bonding adhesion strength was quantified using a scratch testing technique, and the relationships between the critical loads measured and the areal surface parameters, as well as discussion of the underlying mechanisms, are presented in this report. Statistical analysis was employed to identify the most relevant areal parameters that may be used for prediction of the copper/glass bonding strength and for design of adhesion promoting surface textures. The experimental results suggest that the most significant areal surface texture parameters to consider are $S q, S d q, S d r, S x p, V v, V m c$, and $V v c$, and the recommended value range for each parameter for optimal plating adhesion performance is given.
\end{abstract}

Keywords: Surface topography, areal parameters, scratch testing, plating adhesion.

\section{INTRODUCTION}

Glass has been previously explored as an alternative substrate material for FR-4 printed circuit boards, with copper being mostly chosen to be deposited on the glass to act as interconnect metallization due to its low electrical resistivity, high thermal conductivity, good mechanical properties, and high electromigration resistance [1]. When considering glass as an electronics substrate, then electroless plating is required to deposit the conductive tracks and features (circuit layup) onto the insulating glass substrate surface [2]. However, it is difficult for metallization on the smooth glass surface, mainly due to physical, chemical and mechanical mismatch between the metal coating and glass substrate unless the glass is textured in some manner [3]. If chemical, mechanical or laser ablation based texturing is applied, then the finished surfaces require characterizing using surface measurement techniques in order to understand and optimize surface modification process parameters.

One of the most significant issues that defines durability of the copper plating is the quality of bonding of the copper to the glass substrate, with adhesive bond strength being a function of electrochemical and surface mechanical components, noting that work has previously started to examine the electrochemical components of glass/copper adhesion [4], [5]. The complex relationship between roughness and adhesion is not unique to the issue of depositing copper on glass and generically has interested different industrial sectors for more than 50 years.

During this time span, completing surface texture characterization and measurement, analysis and quantification by the majority of researchers has typically been limited to $2 \mathrm{D}$ surface texture profile parameters as defined in ISO 4287 [6], even though these parameters provide a limited understanding of the manufactured surface. Surface roughness average (arithmetical mean deviation) $(R a)$ is a familiar profile parameter and has previously been used to characterize the effect of substrate surface roughness and coating thickness on the adhesion behavior of TiN film deposited on steel substrates [7], as well as being employed to define a linear relationship with average cleavage strength, which was also a function of linear profile length Rlo [8]. Existing work has also investigated the relationships between adhesive strength, surface texture and wettability for plating martensitic stainless steel for adhesion, concluding that 
(understandably) wettability was improved and adhesive strength increased when surface roughness $R a$ increased [9], [10].

However, additional 2D parameters have also been nominated as being of potential use when trying to understand issues of plating adhesion and bond strength. This is illustrated by adhesive bond strength of medium density fiberboard panels and the effects of postmanufacture heat-treatment on surface roughness, characterized by $R a, R y$ (legacy parameter from an earlier version of ISO 4287), and $R z$ [11]. The Root Mean Square deviation of the assessed profile $(R q)$ was used to understand the distribution of the adhesion force of a sapphire particle onto rough alumina substrates [12] and in a separate study the adhesive strength of copper/alumina due to the contact area at the interface [13].

These research outputs (and others) demonstrate that whilst offering some insight and understanding of the role being played by the substrates and surface features, traditional 2D surface texture parameters computed on line profile data are limited from a statistical point of view because the values obtained are sensitive to: the sampled region, direction of profile, and even spurious features. In addition, it has become routinely clear that one profile parameter in isolation cannot fully describe the relationships between surfaces and bonding characteristics, and consequently multiple and simultaneous parameters would be more effective.

The more recent advent of 3D Areal parameters as defined by ISO 25178-2 [14] linked to the maturation of 3D measurement techniques, has opened up more opportunity for describing the cause and effect plating mechanics and more specifically of copper/glass bonding characteristics. Areal parameters make full use of the areal data set and capture much more of the complexity of surfaces [15]. Some of the areal parameters are mathematical extensions of their $2 \mathrm{D}$ forebears, hence it is not surprising that the areal surface roughness parameter $S a$ was soon explored to attempt to characterize the roughness of adhesion between glass/epoxy and carbon/epoxy composites [16], [17], showing the correlation between the surface characteristics of composite materials and adhesion performances of corresponding surface assemblies. The interest of surface treatment to increase surface roughness was discussed in terms of wettability.

In slightly more recent times, other science areas have caught up with the existence of areal parameters and their potential to help understand adhesion, with a range of $S$ parameters being in the biomedical sciences used to measure the adhesion and proliferation of fibroblasts and bacterial strains [18] as well as soft tissue attachment to titanium abutments (noting that this work also considered profile parameters) [19]. In addition to being explored within civil engineering applications - again attempting to correlate surface topography to adhesion or concrete and epoxy layers [20]-[22] as well as steel-epoxy adhesion interfaces [23], and initial work on the bond strength of copper/glass [24].

However, the quantified influence of surface topography has typically been considered in a superficial manner with limited published evidence for the use of areal parameters in the context of plating adhesion. The challenge even with the extended analytical capacity of the areal parameters is that yet again - one areal parameter (such as $S a$ ) is not only inadequate to fully understand the complex surface texture but it is also difficult to understand the influence of areal parameters upon the whole surface properties towards adhesive performance.

The work reported concentrates on defining and applying areal surface texture parameters for functional characterization of structured surfaces with respect to adhesion, allowing a systematic study of the effect of surface topography on electroless plated copper adhesion. The aim is to illustrate that areal parameters can be used in a predictive manner to show the correlation between the surface characteristics of substrate materials and adhesion performances - thus linking to bond strength measurement.

\section{GLASS PROCESSING AND MEASUREMENT}

The use of lasers for the machining of traditional circuit board substrates such as FR-4 with features such as microvias are now being offered as commercial equipment solutions. In addition, there has been a significant increase of research into the application of lasers for ablative machining of various substrates with the advent of controllable materials processing picosecond and femtosecond lasers [25], [26].

Previous work (specifically in the context of circuit layup) has already investigated the feasibility of the $248 \mathrm{~nm}$ excimer laser in the laser structuring of fine circuit lines and micro-via features on printed circuit boards [1], [27]. These lasers are capable of making microstructures with feature size on the order of $1 \mu \mathrm{m}-100 \mu \mathrm{m}$ and are applicable for glass-based materials [28]. In this study reported here, commercially available CMG glass supplied by Qioptiq Ltd was chosen as a substrate material due to the similarity of thermal expansion coefficient to silicon and susceptibility to machining by ultraviolet (UV) laser radiation. CMG glass is a borosilicate type glass with a nominal cerium dioxide content and highly absorbing to light with a wavelength shorter than approximately $320 \mathrm{~nm}$. The glass sheets were supplied as square samples with dimensions $40 \mathrm{~mm} \times$ $40 \mathrm{~mm}$ with thicknesses of $100 \mu \mathrm{m}$ and $500 \mu \mathrm{m}$.

A Krypton Fluoride $(\mathrm{KrF})$ Excimer laser (model EMG 203, Lambda Physik) operating at $248 \mathrm{~nm}$ was used for machining. The laser system parameters included: output pulse energy $(200 \mathrm{~mJ}-250 \mathrm{~mJ})$, pulse duration $(20 \mathrm{~ns})$, repetition rate $(5 \mathrm{~Hz}-25 \mathrm{~Hz})$, attenuator position (0.2-0.9), and shots per area (5-200), and were varied and optimized according to the surface topography and adhesion performance of the samples. The parameters chosen for use in this work were identified to produce an energy density of $2.4 \mathrm{~J} / \mathrm{cm}^{2}$ at the workpiece surface when operated with a pulse repetition rate of $10 \mathrm{~Hz}$.

Excimer lasers can generate micro-scale pattern array structures on glass by using a mask projection and dragging process [29], [30], this being a common and modern commercial solution. Various metal masks with square, circular and triangular apertures were placed in the laser 
path to tailor the size and the shape of laser beam projected onto the glass substrates. Complex micro-scale structures were typically produced in two steps. Firstly, the glass work piece was mounted on the computer numerically controlled (CNC) work stage to form a groove contour along one direction, with step-wise repetition of the process forming overlapping grooves. Secondly (and where necessary), grooves were machined orthogonally to the initially machined grooves, creating segmented periodic structures.

A Zygo NewView 5000 coherence scanning interferometer (CSI) system (calibrated and traceable to the NIST defined metre standard) was used to measure the etch depth and areal surface topography of each machined circular feature or well. The surface roughness measurement was processed and analysed using the TalyMap Platinum v5.1 (DigitalSurf Mountains) surface texture processing software compliant to ISO 25178-2. Typically, a $10 \times$ objective lens and $1 \times$ optical zoom were used to provide a lateral field of view of approximately $700 \mu \mathrm{m} \times 500 \mu \mathrm{m}$, which was predominately used as the sampling area in this research.

Typical structured surface topographies produced by laser machining with different masks are shown in Fig.1.

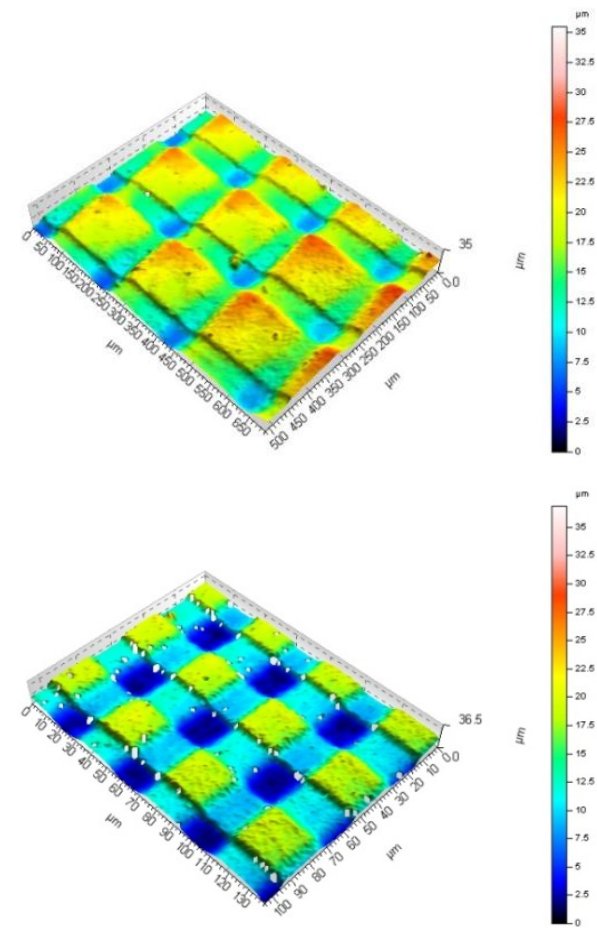

Fig.1. Two examples of structured surfaces created by excimer laser machining showing sequentially ablated grooves in a crosshatched matrix.

\section{SCRATCH TESTING ON PLATED GLASS}

Electroless copper plating was used to metallise the textured glass substrate because the glass substrate is nonconductive. The Circuposit ${ }^{\mathrm{TM}}$ 3350-1 electroless copper chemicals provided by Rohm and Haas Electronic Materials Europe Ltd were used in this study, developed for printed wiring board applications.
Scratch testing was chosen as the most effective quantitative traditional assessment of adhesion strength between copper coating and the $\mathrm{CMG}$ glass substrates. The ST 3001 scratch testing system at the UK National Physical Laboratory (NPL) with increasing ramp loading (calibrated by a certified reference load cell) was used in this research. A Rockwell C diamond stylus was used with a $200 \mu \mathrm{m}$ tip radius and a cone apex angle of $120^{\circ}$. Ramp loading was increased from a minimum load of $1 \mathrm{~N}$ to $15 \mathrm{~N}$ with 30 steps determined through a series of preliminary experiments.

The optical coordinate measuring machine is a reliable method for the detection of surface damage. This technique allows the user to visually differentiate between cohesive failure within the coating and adhesive failure at the interface of the coating/substrate system. Examples of test scratches on copper coated glass samples can be seen in Fig.2. (OGP Flash 200 optical coordinate measuring machine images (CMM)) and Fig.3. (scanning electron microscope (SEM) images). Failure points along the scratch are typically distinguished by backlighting penetrating through the copper layer and are easier to recognize using white light techniques compared to SEM images. Via the scratch testing results, knowledge of the sample stress state leading to delamination failure is available through direct measurement of the distance from the loading start point to coating penetration and by theoretical calculation. In this case, the value of the critical load $L c(\mathrm{~N})$ can be calculated using equation (1):

$$
\begin{aligned}
& L c=F_{\text {Start load }}+\left(F_{\text {End load }}-F_{\text {Start load }}\right) \times L_{\text {Delamination length / }} L_{\text {Scratch }} \\
& \text { length }
\end{aligned}
$$

Where $F_{\text {Start load }}=1.0 \mathrm{~N}, F_{\text {End load }}=15.0 \mathrm{~N}, L_{\text {Scratch length }}=$ $5.0 \mathrm{~mm}$, so the calculation of $L c$ can be expressed as equation (2):

$$
\begin{aligned}
& L c=1.0+(15.0-1.0) \times L_{\text {Delamination length }} / 5.0=1+2.8 \times \\
& L_{\text {Delamination length }}
\end{aligned}
$$

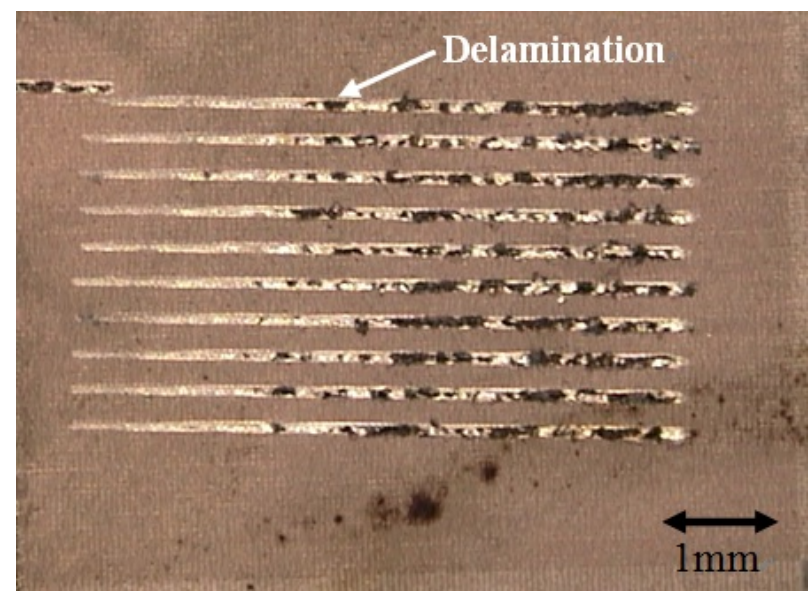

Fig.2. Optical CMM images for scratches of copper coating on CMG glass showing onset of delamination. 


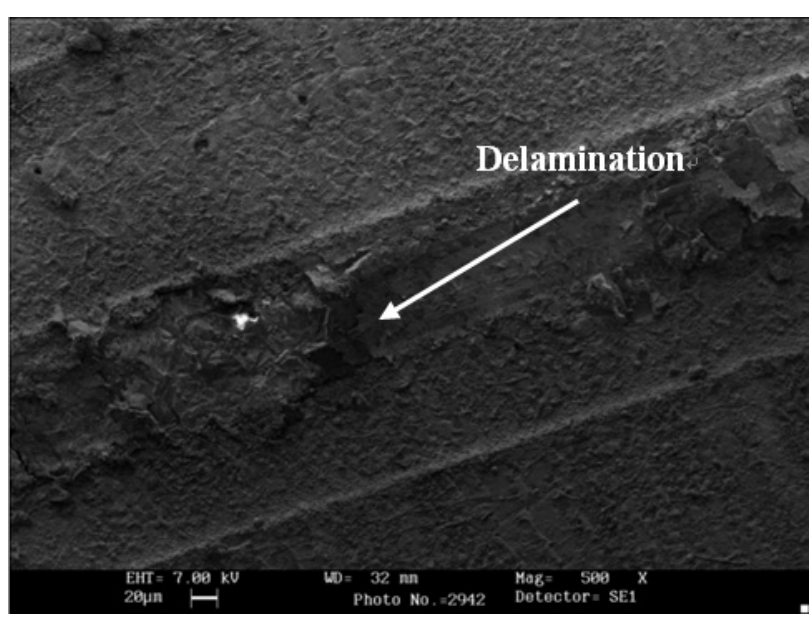

Fig.3. SEM images for scratches of copper coating on CMG glass showing onset of delamination.

\section{CORRELATION OF AREAL PARAMETERS WITH CRITICAL LOADS}

A $25 \mu \mathrm{m}$ L-filter was employed to extract the microroughness component and acquire an S-L surface. Microroughness on the glass surface is an inherent function of the stochastic nature of the excimer laser wavefront spatial characteristics, and has been evaluated in its own right with glass samples featuring micro-roughness, but no structure. A compromise selection of the nesting indices for S-filter was determined based on the specific pitches of samples to remove all the unwanted large scale noisy components and keep both micro-roughness components and structural components. Furthermore, process consideration of this data (and other data sets) confirmed the requirement to investigate a number of potential failure modes for data processing investigation. This is because the failure mode analysis is prone to premature and/or random copper bond failure, which is illustrated in the two sets of tracks in Fig.4. (black points along the scratches indicate possible localized failure points).

A common feature of existing scratch testing analysis is the description of the coating removal process in terms of an interfacial shear force, and coating failure is considered to occur when a critical load is reached. However, sometimes coating failure commenced at micro pattern edges because of stress concentrations and edge effects caused by stylus tip penetration. Alignment of the scratch testing stylus with the sample was a manual process and therefore difficult to guarantee alignment with the preferential direction of the structured surface. Fig.4. shows slight misalignment of the scratch track causing a prolonged impact on a structure edge along each track (half way along each track - seen as an initial black line across the scratch). Once the stylus leaves the feature edge and continues along the structure surface, plating failure is no longer observed, until the stylus load reaches the critical value at the end of each scratch.

Consequently, analysis of the data has led to the development within this research of three failure mode descriptors as being more representative of bulk copper adhesion (and potential user application scenarios) as shown in Fig. 5.:

- Mode 1 - simple - the point where the first failure occurs on the plated surface (as illustrated in Fig.5.);

- Mode 2 - consecutive - the point where three consecutive observable individual failure points are identified within a predefined length of $0.5 \mathrm{~mm}$ (typically a higher critical load compared to the first definition);

- Mode 3 - continuous - the point where continuous delamination occurs for a minimum length of $0.2 \mathrm{~mm}$ (typically the highest critical load compared to the first two definitions).

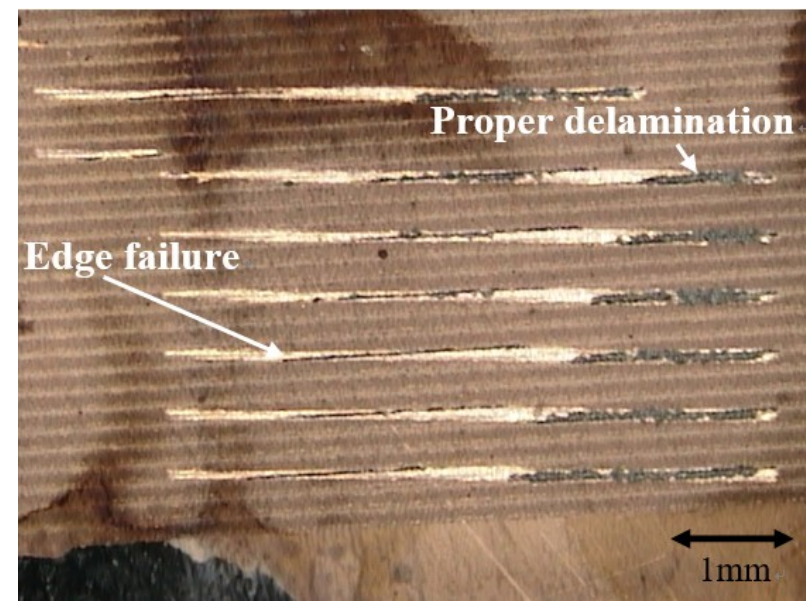

Fig.4. Premature plating failure as a function of stylus / discontinuity impact.

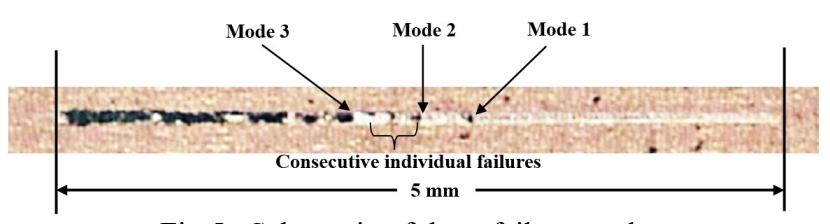

Fig.5. Schematic of three failure modes.

In this study, the Mode 3 criterion was used because it represented catastrophic failure of the copper, when assessing the adhesive bond strength from scratch testing of electroless coated glass substrates and was regarded as the most rigorous and consistent test for copper plating failure.

Modes 1 and 2 were susceptible to spurious, nonrepresentative plating failure as a function of edge effect stress concentrations in the case of Mode 1, and localized random defects in the case of Mode 2.

\section{RESULTS AND DISCUSSION}

Areal parameters (including field and feature parameters) for samples were calculated according to ISO 25178-2. Two correlation coefficients have been employed to quantify the strength of relationship between the areal parameter values and the mean critical load value. 
- Pearson product moment correlation coefficient $(\rho)$ provides a measure of the strength of the linear dependence between two variables $(+1.0$ to -1.0$)$.

- Spearman's rank correlation coefficient $(r)$ provides a non-parametric measure of the statistical dependence between two variables $(+1.0$ to -1.0$)$.

In this manner, it has been possible to select areal parameters from ISO 25178-2 that from an engineering and surface topography sense would most probably be useful in describing the key surface features. Then categorize each areal parameter in terms of the strength of correlation with the critical load values, being mindful of the definition and relevance of each parameter. In each analysis case, the quality of correlation has increased as the failure mode has been changed from simple, to consecutive, to continuous. Fig. 6 shows the correlations for areal parameters that have demonstrated the strongest behavioural relationships with the copper/glass critical load values, with the correlation coefficient values summarized in Table 1.

Table 1. Correlation coefficients defining the relationship between areal parameters and scratch testing critical load.

\begin{tabular}{|c|c|c|}
\hline Areal parameters & $\begin{array}{c}\text { Pearson } \\
(\boldsymbol{\rho})\end{array}$ & $\begin{array}{c}\text { Spearman's } \\
(\boldsymbol{r})\end{array}$ \\
\hline$S q$ & -0.74 & -0.71 \\
\hline$S d q$ & -0.69 & -0.66 \\
\hline$S d r$ & -0.64 & -0.61 \\
\hline$S x p$ & -0.66 & -0.63 \\
\hline$V v$ & -0.74 & -0.72 \\
\hline$V m c$ & -0.77 & -0.74 \\
\hline$V v c$ & -0.73 & -0.70 \\
\hline
\end{tabular}

Fig.6. indicates there are general marked decreasing trends with critical load increase. $S q$ is the root mean square height of the scale limited surface, and has direct analogies to the profile $R$ and $W$ two dimensional line profile derived parameters. As one of the height parameters, the $S q$ value depends on height deviation of the surface and is one of the most widely used parameters which indicate surface roughness in a well-known statistical form. For structured surfaces, the exit of the copper is partially blocked by part of the glass substrate from scratch testing. This place in the interface will exhibit the so-called mechanical interlocking effect. The copper in a valley cannot move without plastically deforming such as spalling because of the physical impediment. Plastic deformation acts as an energy absorbing mechanism and the strength of the adhesive bond appears to increase, therefore the critical load from scratch testing is higher. However, if the micro-roughness is too large, shown as the failure start point in Fig.4., it will also cause the random failure through sharp edges effect.

$S d q$ is the root mean square gradient of the scale limited surface within the definition area, and is a hybrid parameter that is generated on the basis of amplitude and spatial information about the surface. The difference of $S d q$ means that not only the size of peaks and valleys is higher in case of a rough surface, but they are steeper as well. A higher $S d q$ value may signify a peaky surface with many sharp edges and this trend shows the same expectation in this study, i.e. sharp edges are susceptible to premature and/or random copper bond failure, resulting in localized failure.

$S d r$ is the ratio of the increment of the interfacial area of a surface over the sampling area. A large value of $S d r$ indicates the significance of either the amplitude, or the spacing, or both. Functionally in adhesion applications, $S d r$ characterizes the real contact area between adherent and adhesive. If interfacial interactions are the basis for adhesion, the sum of those interactions will scale as the area of contact. If the actual area of contact is increased by a large amount, the total energy of surface interaction increases by an amount proportional to the surface area. However, for rough surfaces such as very steep surfaces, the values of the developed interfacial area ratio are usually larger and the critical loads are smaller which means failure occurs earlier. This trend could also be explained by the situation that sharp edges are susceptible to premature and/or random copper bond failure.

Peak extreme height parameter $S x p$ refers to the difference in height between $2.5 \%$ and $50 \%$ of the material ratio. Sxp indicates the depth from the surface material $(2.5 \%)$ to the remaining material area $(50 \%)$ by wear or modification. Fig.6.d) presents a generally decreasing distribution trend and failure occurred earlier with larger $\operatorname{Sxp}$ values. Large scale structures such as deep valleys may result in large $S x p$ value and show weaker plating adhesion because of the sharp edge effect.

$V v(m r)$, the void volume, is the volume of the voids per unit area at a given material ratio calculated from the areal material ratio curve. This parameter refers to the volume of space bounded by the surface texture from a plane at a height corresponding to a chosen " $m r$ " value to the lowest valley. Fig.6.e) shows a clear trend between $V v(m r)$ parameter and critical load. Good plating adhesion occurs with smaller $V v(m r)$ value. It should be noticed that this trend is present for this particular case, i.e. copper plating adhesion strength which is characterized by critical load from delamination failure mode. Surfaces with sharp edges and fluctuated roughness amplitude may cause local random failure which would lower plating adhesion strength.

$\operatorname{Vmc}(p, q)$, the core material volume, is the volume of material comprising the texture between heights corresponding to the material ratio values of $p$ and $q$. This parameter shows how much material is available for load support once the top levels of surfaces are worn away. Fig.6.f) suggests higher critical load occurs with smaller $V m c$ value. This parameter is generated based on the material ratio curve which is termed the total roughness, and $V m c$ indicates a measure of the material forming the surface between various heights which strongly depends on the amplitude of surface roughness. Hence $V m c$ shows a similar trend compared to the micro-roughness characterization $S q$. Moreover, surfaces with extreme valleys result in large $V m c$ values. These extreme valleys from local micro-roughness could be regarded as being equivalent to the large scale 
structured surface edge. From the previous discussion, it is suggested that this could result in failure due to the sharp edge effect.

a)

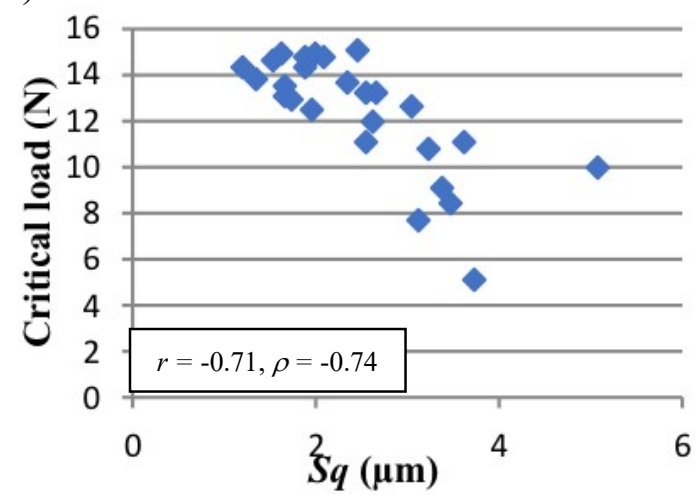

b)

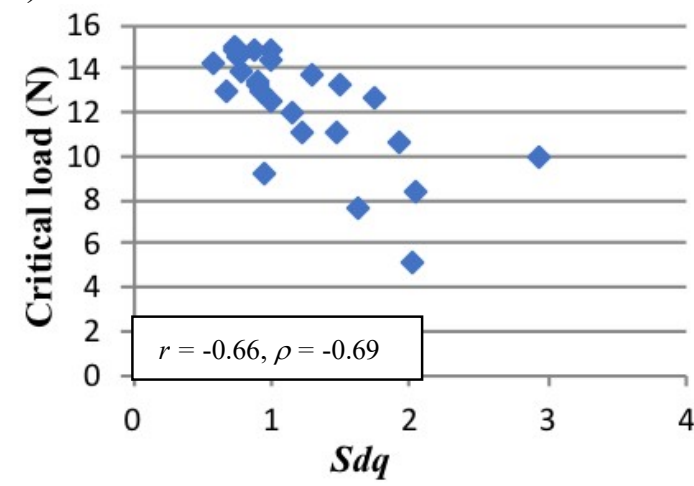

c)

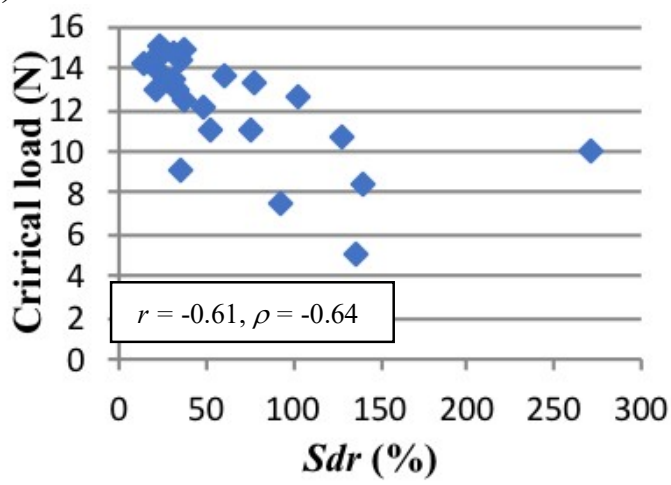

d)

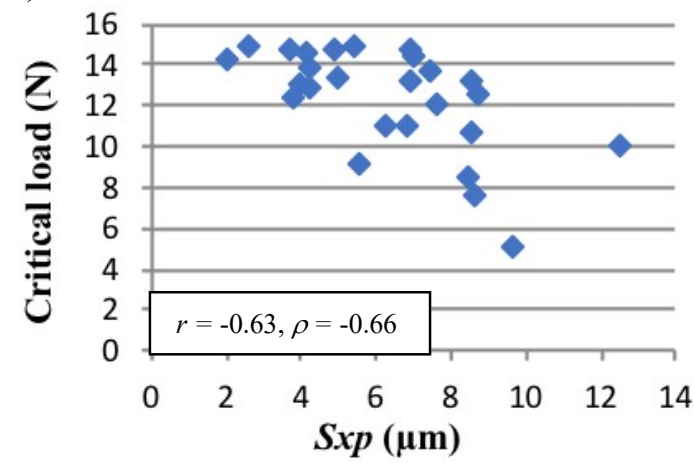

e)

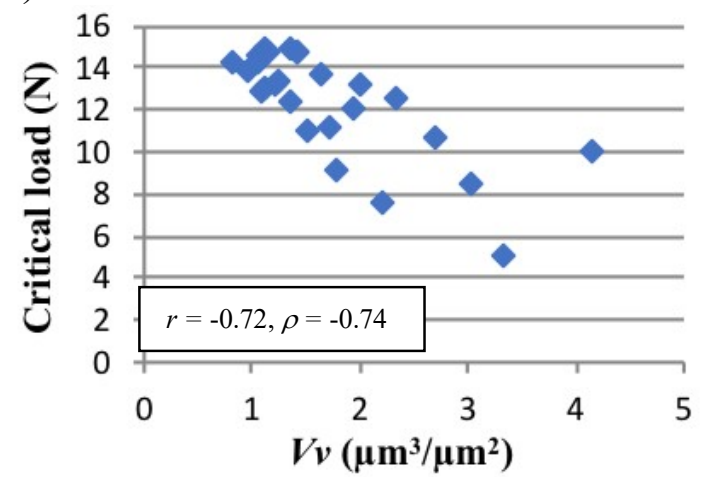

f)

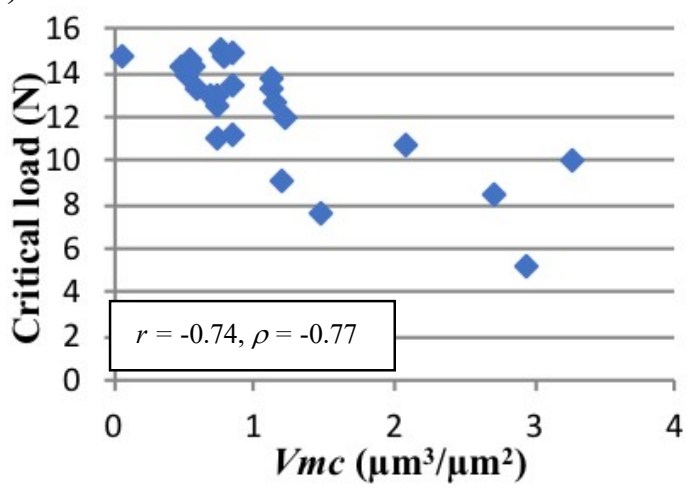

g)

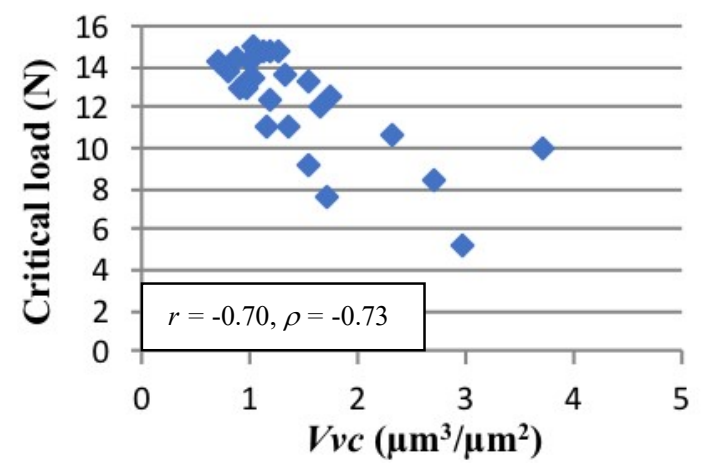

Fig.6. Correlations for areal parameters versus critical load for structured glass. a) $S q$ (S-filter applied), b) $S d q$ (S-filter applied), c) $S d r$ (S-filter applied), d) $\operatorname{Sxp}$ (L-filter applied), e) $V v$ (L-filter applied), f) $V m c$ (L-filter applied), g) $V v c$ (L-filter applied).

$V v c$ is the core void volume of the scale limited surface. This is one of a number of material/void volume parameters derived from the volume information of the areal material ratio curves, with the $V v c$ parameter functionally related to the $10 \%$ to $80 \%$ range of the material ratio. This parameter may provide information about the resulting void volume for fluid entrapment or leakage. It can be seen in Fig.6.g) that there is a clear trend between the $V v c$ parameter and the critical load. Good plating adhesion occurs with smaller $V v c$ value. The reason can also be explained by the interaction of mechanical interlocking effect and sharp edge effect.

The key parameters identified which show the strongest correlations and may functionally be most relevant with respect to copper/glass adhesion for structured surfaces are 
shown in Table 2. [15], which clearly demonstrate potential for predicting surface capability for improved plating characteristics. This set of selected areal parameters is considered to be most likely to allow a comprehensive characterization of the relationship between surface topography and adhesion in this immediate context of electroless copper plating of excimer ablated processed CMG glass substrates.

Table 2. Rank ordered areal parameters.

\begin{tabular}{|c|c|}
\hline $\begin{array}{c}\text { Areal } \\
\text { parameters }\end{array}$ & Recommended value \\
\hline$V m c$ & $0.4 \mu \mathrm{m}^{3} / \mu \mathrm{m}^{2}-1.5 \mu \mathrm{m}^{3} / \mu \mathrm{m}^{2}$ \\
\hline$V v$ & $0.5 \mu \mathrm{m}^{3} / \mu \mathrm{m}^{2}-2 \mu \mathrm{m}^{3} / \mu \mathrm{m}^{2}$ \\
\hline$S q$ & $1 \mu \mathrm{m}-2.5 \mu \mathrm{m}$ \\
\hline$V v c$ & $0.5 \mu \mathrm{m}^{3} / \mu \mathrm{m}^{2}-1.5 \mu \mathrm{m}^{3} / \mu \mathrm{m}^{2}$ \\
\hline$S d q$ & $0.5-1$ \\
\hline$S x p$ & $0.5 \mu \mathrm{m}-5 \mu \mathrm{m}$ \\
\hline$S d r$ & $10 \%-50 \%$ \\
\hline
\end{tabular}

For the parameters identified in Table 2., there are strong trends being shown between critical load and the respective areal parameter. Furthermore, it is recognized that these results can be reinforced by the graphical and numerical comparisons with data points from glass samples, without any delamination during the scratch testing within the load between $1.0 \mathrm{~N}$ and $15.0 \mathrm{~N}$. It should be noted that the maximum load applied by the scratch testing equipment was 15.0 N, this being determined by catastrophic failure of the glass substrate at greater loads. If no copper plating failure was observed during a scratch test, then the default critical load value was regarded as $15.0 \mathrm{~N}$, however, this non-failed data was excluded from the data processing shown in the previous graphs. Consequently, it is possible that some of the copper/glass samples may have higher bond strengths than $15.0 \mathrm{~N}$, but it has not been possible to quantify this unless thicker CMG glass substrates are subsequently used to investigate this issue.

\section{CONCLUSIONS}

This study has specifically considered the mechanical contribution of the CMG glass surface texture (produced through the use of Excimer laser ablation) to the electroless copper/glass adhesive bond, and attempting to isolate ISO 25178-2 areal surface texture parameters that can describe these surfaces, establishing the statistical quality of correlation between the critical load values and the associated areal parameters. Two correlation coefficients (Spearman and Pearson correlation ranking coefficients) have been employed to quantify the strength of relationship between the areal parameter values and the associated critical loads. Therefore, adhesive bond strength and areal parameters have been identified as showing a strong correlation with critical load. The parameters $S q, S d q, S d r$, $S x p, V v, V m c$, and $V v c$ are regarded as having the potential for appropriately describing the glass surfaces in the context of electroless copper bonding, on the basis of the correlation results within the data sets, but also with reference to their descriptions and mathematical functions as identified in ISO 25178:2. It is further noted that these parameters have differing mathematical derivations - some with potentially more of a statistical bias than others. It is important that for further use of such parameters, functional understanding of any link between the definition of any one parameter and the surface science or engineering is explored.

\section{ACKNOWLEDGEMENT}

This research is collaboration between Loughborough University (UK) and the UK National Physical Laboratory. The research has been part funded by the EPSRC 3DMintegration Grand Challenge project, the Wolfson School of Mechanical, Electrical \& Manufacturing Engineering, and UK NMO Engineering \& Flow Metrology Programme. The authors would also like to thank Qioptiq Ltd for the supply of CMG glass.

\section{REFERENCES}

[1] Hutt, D.A., Williams, K., Conway, P.P., Khoshnaw, F.M., Cui, X., Bhatt, D. (2007). Challenges in the manufacture of glass substrates for electrical and optical interconnect. Circuit World, 33 (1), 22-30.

[2] Cui, X., Hutt, D.A., Conway, P.P. (2012). Evolution of microstructure and electrical conductivity of electroless copper deposits on a glass substrate. Thin Solid Films, 520 (19), 6095-6099.

[3] Hidai, H., Tokura, H. (2001) Direct laser writing of aluminum and copper on glass surfaces from metal powder. Applied Surface Science, 174 (2), 118-124.

[4] Charbonnier, M., Romand, M. (2002). Tin-free electroless metallization of glass substrates using different PACVD surface treatment processes. Surface \& Coatings Technology, 162, 19-30.

[5] Wang, M.-W., Liu, T.-Y., Pang, D.-C., Hung, J.-C., Tseng, C.-C. (2014). Inkjet printing of a $\mathrm{pH}$ sensitive palladium catalyst patterns of ITO glass for electroless copper. Surface \& Coatings Technology, 259, 340345.

[6] ISO - International Organization for Standardization. (1997). Geometrical Product Specifications (GPS) Surface texture: Profile method - Terms, definitions and surface texture parameters. ISO 4287:1997.

[7] Takadoum, J., Bennani, H.H. (1997). Influence of substrate roughness and coating thickness on adhesion, friction and wear of TiN films. Surface \& Coatings Technology, 96 (2-3), 272-282.

[8] Shahid, M., Hashim, S.A. (2002). Effect of surface roughness on the strength of cleavage joints. International Journal of Adhesion and Adhesives, 22 (3), 235-244. 
[9] Minaki, K., Kitajima, K., Minaki, K., Izawa, M., Tosha, K. (2005). Improvement of surface texture of stainless steel by utilizing dry blasting -2 nd report: Effect of blasting conditions on wettability. Key Engineering Materials, 291-292, 265-270.

[10] Minaki, K., Kitajima, K., Minaki, K., Izawa, M., Tosha, K. (2007). Improvement of surface texture of stainless steel by utilizing dry blasting - 3rd report: Effect of blasting surface texture on adhesion of plating. Key Engineering Materials, 329, 353-358.

[11] Ayrilmis, N., Winandy, J.E. (2009). Effects of post heat-treatment on surface characteristics and adhesive bonding performance of medium density fiberboard. Materials \& Manufacturing Processes, 24 (5), 594599.

[12] Audry, M.C., Ramos, S., Charlaix, E. (2009). Adhesion between highly rough alumina surfaces: An atomic force microscope study. Journal of Colloid and Interface Science, 331, 371-378.

[13] Ju, D.L., Susan, Y.S.Y., Daniel, M.W., Leong, K.C., Wong, C.C. (2013). Surface roughness effect on copper-alumina adhesion. Microelectronics Reliability, 53 (9-11), 1548-1552.

[14] ISO - International Organization for Standardization. (2012). Geometrical product specifications (GPS) Surface texture: Areal - Part 2: Terms, definitions and surface texture parameters. ISO 25178-2:2012.

[15] Petzing, J.N., Coupland, J.M., Leach, R. (2010). The Measurement of Rough Surface Topography using Coherence Scanning Interferometry. NPL Measurement Good Practice Guide 116. National Physical Laboratory.

[16] Bénard, Q., Fois, M., Grisel, M. (2005). Influence of fibre reinforcement and peel ply surface treatment towards adhesion of composite surface. International Journal of Adhesion and Adhesives, 25 (5), 404-409.

[17] Bénard, Q., Fois, M., Grisel, M. (2006). Surface treatment of carbon/epoxy and glass/epoxy composites with an excimer laser beam. International Journal of Adhesion and Adhesives, 26 (7), 543-549.

[18] Pacha-Olivenza, M.A., Tejero, R., FernandezCalderon, M.C., Anitua, E., Troya, M., GonzálesMartin, M.L. (2019). Relevance of topographic parameters on the adhesion and proliferation of human gingival fibroblasts and oral bacterial strains. BioMed Research International, 2019, 8456342.

[19] Brunello, G., Brun, P., Gardin, C., Ferroni, L., Bressan, E., Meneghello, R., Zavan, B., Sivolella, S. (2018). Biocompatibility and antibacterial properties of zirconium nitride coating on titanium abutments: An in vitro study. PLoS ONE, 13 (6), e0199591.

[20] Hoła, J., Sadowski, L., Reiner, J., Stach, S. (2015). Usefulness of 3D surface roughness parameters for nondestructive evaluation of pull-off adhesion of concrete layers. Construction and Building Materials, 84, 111-120.
[21] Sadowski, L., Czarnecki, S., Hoła, J. (2016). Evaluation of the height $3 \mathrm{D}$ roughness parameters of concrete substrate and the adhesion to epoxy resin. International Journal of Adhesion and Adhesives, 67, 3-13.

[22] Sadowski, L., Hoła, J., Czarnecki, S., Wang, D. (2018). Pull-off adhesion prediction of variable thick overlay to the substrate. Automation in Construction, $85,10-23$.

[23] van Dam J.P.B., Abrahami, S.T., Yilmaz, A., Gonzalez-Garcia, Y., Terryn, H., Mol, J.M.C. (2020). Effect of surface roughness and chemistry on the adhesion and durability of a steel-epoxy adhesive interface. International Journal of Adhesion and Adhesives, 96, 102450.

[24] He, B., Petzing, J., Webb, P., Leach, R. (2015). Improving copper plating adhesion on glass using laser machining techniques and areal surface texture parameters. Optics and Lasers in Engineering, 75, 3947.

[25] Abere, M.J., Zhong, M., Krüger, J., Bonse, J. (2016). Ultrafast laser-induced morphological transformations, MRS Bulletin, 41, 969-974.

[26] Canfield, B.K., Costa, L., Rajput, D., Terekhov, A., Lansford, K., Hofmeister, W.H., Davis, L.M. (2020). Machining of micrometer-scale high aspect ratio features with single femtosecond laser pulses. Journal of Laser Applications, 32 (3), 032021.

[27] Leach, R.K. (2001). The measurement of surface texture using stylus instruments. NPL Measurement Good Practice Guide 37. National Physical Laboratory.

[28] Zhang, B., Yung, K.C. (2007). Feasibility of the 248 $\mathrm{nm}$ Excimer laser in the laser structuring of fine circuit lines on printed circuit board. The International Journal of Advanced Manufacturing Technology, 33, 1149-1158.

[29] Chen, Y.T., Ma, K.J., Zhou, J.G., Tseng, A.A. (2005). Excimer laser ablation of glass-based arrayed microstructures for biomedical, mechanical, and optical applications. Journal of Laser Applications, 17 (1), 38-46.

[30] Wang, S.Y. (2005). A computer simulation for maskshape effect in the fabrication of an aspheric micro lens array by using a dragging process with excimer laser. Journal of Micromechanics and Microengineering, 15, 1310-1316.

Received November 11, 2020 Accepted February 28, 2021 